\title{
Survival Comparison of Patients Undergoing Secondary Aortic Repair
}

\author{
Dean J. Yamaguchi, ${ }^{1}$ Thomas C. Matthews, ${ }^{2}$ Marjan Mujib, ${ }^{2}$ Marc A. Passman, \\ Mark A. Patterson, ${ }^{2}$ Bart R. Combs, ${ }^{2}$ and William D. Jordan Jr. ${ }^{2}$ \\ ${ }^{1}$ Department of Cardiovascular Sciences, East Carolina University Brody School of Medicine, Greenville, NC 27834, USA \\ ${ }^{2}$ Section of Vascular Surgery and Endovascular Therapy, University of Alabama at Birmingham, Birmingham, AL 35294, USA \\ Correspondence should be addressed to Thomas C. Matthews; tcmatthews@uabmc.edu
}

Received 21 September 2014; Accepted 5 March 2015

Academic Editor: Roberto Pola

Copyright (C) 2015 Dean J. Yamaguchi et al. This is an open access article distributed under the Creative Commons Attribution License, which permits unrestricted use, distribution, and reproduction in any medium, provided the original work is properly cited.

\begin{abstract}
Introduction. Infrarenal abdominal aortic aneurysm (AAA) repair warrants lifelong surveillance. Secondary aortic intervention (SAI) outcomes may be affected by the therapeutic approach. We compared short- and long-term mortality in patients who underwent SAIs after initial aortic repair, either endovascular (EVAR) or open. Methods. Patients who underwent AAA repair between 1986 and 2010 were retrospectively identified in a vascular surgery database as well as those who underwent SAIs. All-cause mortality and Kaplan-Meier survival curves were calculated. Results. We identified 149 patients who underwent either open AAA repair or EVAR followed by open or endovascular SAI. Seventy-seven patients (51.7\%) underwent initial EVAR while 72 patients (48.3\%) underwent open repair. Sixty (78\%) initial EVAR patients underwent secondary EVAR while $17(22 \%)$ patients had an open SAI. Initial open repair patients were evenly distributed between EVAR and open SAIs. Compared to EVAR, patients who underwent initial open repair had longer intervals between primary aortic interventions (PAIs) and SAIs. Multivariable-adjusted all-cause mortality was significantly higher for patients who underwent initial open AAA repair followed by EVAR when compared to patients who underwent endovascular PAI and SAI. Conclusion. Long-term mortality in patients with infrarenal aortic aneurysms who require SAI may be improved by an EVAR-first algorithm.
\end{abstract}

\section{Introduction}

EVAR is an acceptable therapeutic approach to anatomically appropriate infrarenal abdominal aortic aneurysms. However, even with the short-term benefits such as shorter hospital stays, there may be a greater need for future interventions. Approximately $12 \%$ to $20 \%$ of patients who undergo EVAR require secondary procedures, mandating lifelong surveillance for the development of stent graft fracture, migration, or even the expansion of aneurysms outside the treated area [1-4]. Moreover, one-third of patients evaluated for thoracoabdominal aneurysm repair have already undergone a previous operation for aortic aneurysm disease, most often repair of an infrarenal AAA [5]. Both the EVAR1 and DREAM trials highlight the decreasing survival advantage of EVAR over time due to subsequent findings necessitating secondary intervention [6-9]. Our institutional experience has demonstrated improved long-term survival amongst patients who undergo EVAR as opposed to open infrarenal AAA repair. However, we have discovered that EVAR is associated with more secondary interventions while patients who undergo open repair experience greater nonvascular morbidity [4]. Laheij et al. have published their experience of secondary interventions in patients with ruptured and nonruptured AAA after initial EVAR, supporting an endovascular approach for subsequent interventions [3]. However, while endovascular approaches may be feasible in most patients requiring a secondary intervention, it remains unclear as to how they affect mortality. We propose that the initial surgical approach to the management of infrarenal AAA, either open or endovascular, influences subsequent short- and long-term mortality for those patients who require secondary intervention. Furthermore, the natural history of aortic degeneration after initial surgical intervention may be altered by the type 
of aortic repair. As a consequence, the purpose of this study is to compare the mortalities of patients who underwent secondary aortic procedures after initial EVAR or open infrarenal abdominal aortic aneurysm repair.

\section{Materials and Methods}

Between 1986 and 2010, 2256 patients were identified who underwent either open $(N=1012)$ or endovascular $(N=$ 1244) abdominal aortic aneurysm repair. Type of repair was determined based upon aortic anatomy appropriate for EVAR as well as the patient's medical fitness for open aortic operation as determined by the treating surgeon. Endovascular abdominal aortic aneurysm repairs were performed according to instructions for use (IFU) for each device used. To further characterize the natural history of aortic degeneration after initial aneurysm repair, patients who underwent a second intervention along any part of the aorta from the subclavian artery to the iliac bifurcation were included. Follow-up information was obtained from hospital chart review, review of a prospectively maintained vascular surgery database, and query of the social security death index. In this population, we further determined those patients who subsequently underwent a secondary aortic intervention $(N=149)$, either open or endovascular stent graft repair. We divided these patients into four categories: those who had a primary endovascular procedure followed by secondary endovascular procedure were denoted as Endo-Endo. Similarly Endo-Open, OpenEndo, and Open-Open categories were constructed based on the primary and secondary aortic procedures.

The primary endpoint included all-cause mortality during a mean follow-up period of two years after the second procedure. We used chi-squared and ANOVA tests to compare demographics and baseline characteristics between the four aortic procedure groups. Associations of various aortic procedures with all-cause mortality were determined by KaplanMeier survival analysis and Cox proportional hazard models. The Endo-Endo category was used as the reference category, and dummy variables were used for Endo-Open, Open-Endo, and Open-Open categories. Multivariate analysis adjusted for age at secondary aortic procedure, gender, race, duration between primary and secondary aortic procedure, coronary artery disease, hypertension, hyperlipidemia, stroke, obesity, and diabetes mellitus was then performed. We confirmed the assumption of proportional hazards by a visual examination of the $\log$ (minus $\log$ ) curves. All statistical tests were evaluated with the use of two-tailed 95\% confidence level, and tests with $P<0.05$ were considered significant. Data analyses were performed with the use of SPSS for Windows, release 20, 2011 (SPSS Inc., Chicago, IL).

\section{Results}

During the 25-year study period, 149 patients (6.6\%) among 2256 abdominal aneurysm repairs underwent secondary aortic procedures. As depicted in Figure 1, of these 149 patients, 77 (51.7\%) underwent initial EVAR while 72 patients $(48.3 \%)$ underwent open AAA repair. Sixty initial EVAR patients

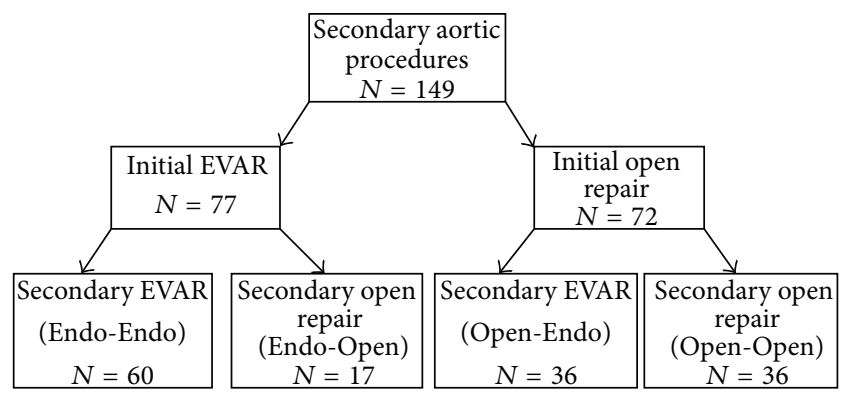

FIGURE 1: Division of patients who underwent initial EVAR or open AAA repair followed by subsequent secondary aortic intervention.

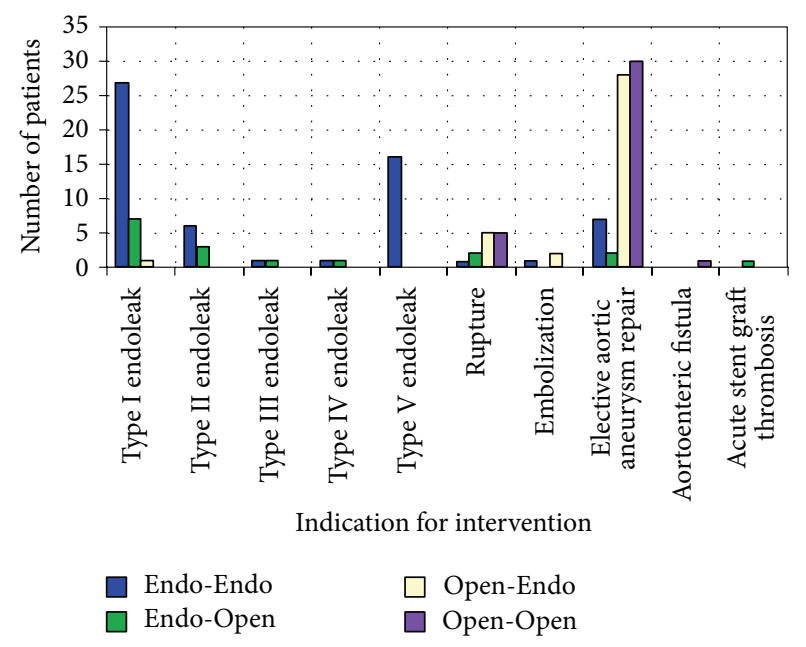

FIGURE 2: Clinical indications for secondary aortic interventions.

(78\% of 77) underwent secondary EVAR (Endo-Endo) while 17 patients (22\% of 77 ) had a secondary open aortic procedure performed (Endo-Open). Initial open repair patients were evenly distributed between EVAR (36) (Open-Endo) and open (36) (Open-Open) secondary repair. In the EndoEndo group, endovascular secondary aortic procedures consisted of 54 additional infrarenal endovascular device placement procedures (90\% of 60 ) and 6 TEVAR interventions (10\% of 60 ). Twenty-five TEVAR procedures (69\% of 36 ) were performed in the Open-Endo group. The Endo-Open group included two open thoracoabdominal aortic aneurysm repairs (12\% of 17$)$.

Indications for secondary aortic intervention included endoleak, rupture, embolization, elective repair, aortoenteric fistula, and acute stent graft thrombosis (Figure 2). In the Endo-Endo group, secondary procedures were performed in 27 patients (45\% of 60) for type I endoleak, 6 (10\% of 60 ) for type II endoleak, 1 ( $2 \%$ of 60 ) for type III endoleak, 1 (2\% of 60 ) for type IV endoleak, and 16 (27\% of 60$)$ for type $\mathrm{V}$ endoleak (continued expansion of the aneurysm without a detectable endoleak), all of which required intervention for expanding aneurysm sacs during follow-up surveillance. Elective secondary aortic aneurysm repair (i.e., thoracoabdominal, infrarenal AAA or thoracic aortic aneurysm) was performed in 28 of 36 Open-Endo patients (78\%) and 30 of 
TABLE 1: Baseline characteristics of patients by type of aortic intervention.

\begin{tabular}{|c|c|c|c|c|c|}
\hline & Endo-Endo $(N=60)$ & Endo-Open $(N=17)$ & Open-Endo $(N=36)$ & Open-Open $(N=36)$ & $P$ value \\
\hline Age at first intervention & $69( \pm 9)$ & $68( \pm 7)$ & $60( \pm 14)$ & $63( \pm 11)$ & 0.001 \\
\hline Age at second intervention & $73( \pm 10)$ & $71( \pm 7)$ & $74( \pm 8)$ & $71( \pm 7)$ & 0.404 \\
\hline Female & $7(12)$ & $4(24)$ & $5(14)$ & $7(19)$ & 0.569 \\
\hline Non-white & $6(10)$ & $3(18)$ & $2(6)$ & $6(17)$ & 0.395 \\
\hline $\begin{array}{l}\text { Interval between first and } \\
\text { second intervention (years) }\end{array}$ & $3( \pm 3)$ & $2( \pm 2)$ & $13( \pm 15)$ & $7( \pm 7)$ & $<0.001$ \\
\hline Coronary artery disease & $34(57)$ & $8(47)$ & $20(56)$ & $17(47)$ & 0.767 \\
\hline Stroke & $4(7)$ & $1(6)$ & $3(8)$ & $5(14)$ & 0.636 \\
\hline Diabetes mellitus & $12(20)$ & $1(6)$ & $7(19)$ & $2(6)$ & 0.143 \\
\hline Hypertension & $43(72)$ & $10(59)$ & $27(75)$ & $26(75)$ & 0.672 \\
\hline Hyperlipidemia & $26(43)$ & $6(35)$ & $21(58)$ & $13(36)$ & 0.218 \\
\hline Obesity & $6(10)$ & $3(18)$ & $2(6)$ & $5(14)$ & 0.520 \\
\hline Smoking & $48(80)$ & $10(59)$ & $31(86)$ & $24(67)$ & 0.073 \\
\hline
\end{tabular}

TABLE 2: Association of secondary aortic procedure with long-term mortality.

\begin{tabular}{lcccc}
\hline & & \multicolumn{3}{c}{ HR $(95 \% \mathrm{CI}) ; P$} \\
& Endo-Endo $(N=60)$ & Endo-Open $(N=17)$ & Open-Endo $(N=36)$ & Open-Open $(N=36)$ \\
\hline Mortality, \% & 28.3 & 23.5 & 47.2 & 52.8 \\
Multivariable-adjusted* & 1.00 (Reference) & $0.785(0.266-2.315)$ & $2.039(1.064-3.910)$ & $1.527(0.828-2.817)$ \\
& & $P=0.660$ & $P=0.032$ & $P=0.175$ \\
\hline
\end{tabular}

${ }^{*}$ Model adjusted for age at secondary aortic procedure, gender, race, duration between primary and secondary aortic procedure, coronary artery disease, hypertension, hyperlipidemia, stroke, obesity and diabetes mellitus.

36 Open-Open patients (83\%). Secondary intervention for rupture was employed in one patient (1.7\%) in the Endo-Endo group, two patients $(11.8 \%)$ in the Endo-Open group, and five patients (13.9\%) each in the Open-Endo and Open-Open groups. One patient (3\% of 36) in the Open-Open group underwent secondary intervention for aortoenteric fistula while one patient ( $6 \%$ of 17 ) in the Endo-Open group required a subsequent procedure for acute stent graft thrombosis.

Patients had a mean age of $72( \pm 8)$ years at secondary intervention, $15 \%$ were women and $11 \%$ were nonwhite. Compared with patients who underwent Endo-Endo or EndoOpen procedures, those with Open-Endo or Open-Open procedures had a lower mean age at the time of first procedure (Table 1). Compared to EVAR-first procedures, initial open procedures had a longer interval prior to secondary aortic intervention (Table 1). Indications for secondary aortic intervention included management of endoleaks, more frequently noted in the EVAR-first groups, as well as elective repair of secondary aortic aneurysms, more commonly encountered in the initial open repair groups.

Over a mean follow-up period of two years, $28 \%, 24 \%$, $47 \%$, and $53 \%$ of patients died in the Endo-Endo, EndoOpen, Open-Endo, and Open-Open categories, respectively (Table 2, Figure 3). Patients who underwent initial EVAR (Endo-Endo and Endo-Open) had better survival than their initial open repair counterparts. Specifically, when compared with the Endo-Endo group, adjusted hazard ratios (HRs) and $95 \%$ confidence intervals (CIs) for all-cause mortality

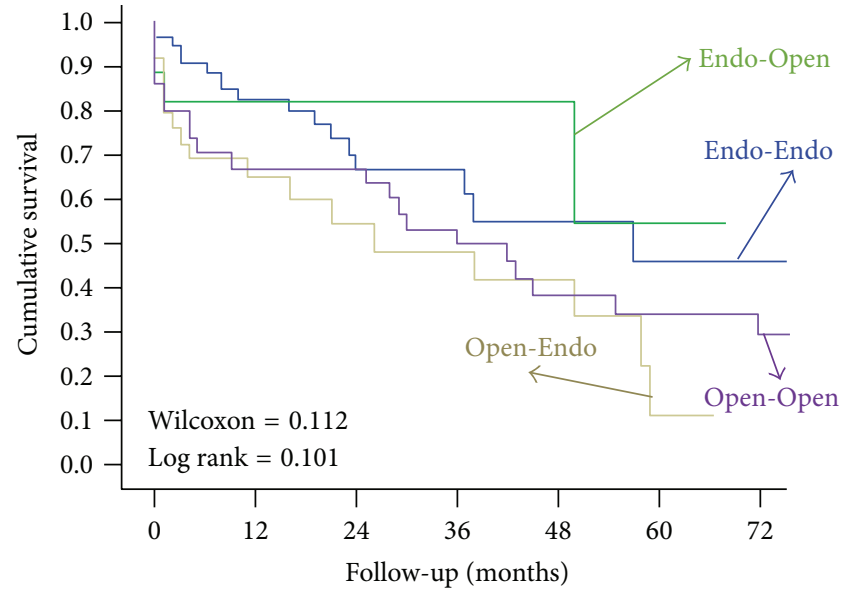

FIGURE 3: Kaplan-Meier plots displaying survival function by secondary conversions after abdominal aortic aneurysm repair.

for those in the Endo-Open, Open-Endo, and Open-Open categories were $0.785(0.266-2.315 ; P=0.660), 2.039$ (1.064$3.910 ; P=0.032)$, and $1.527(0.828-2.817 ; P=0.175)$, respectively (Table 2 and Figure 2).

Further analysis of patients who underwent secondary aortic intervention for emergent indications (i.e., rupture or symptomatic secondary aortic aneurysm) was also performed. Although the number of patients in each subgroup 
was limited, emergent secondary aortic repairs were undertaken in two Endo-Endo patients with one death, three EndoOpen patients with two deaths, nine Open-Endo patients with seven deaths, and ten Open-Open patients with eight deaths. Overall mortality of patients who underwent emergent intervention was $75 \%$ compared with $31 \%$ for those who underwent elective repair.

\section{Discussion}

While there have been recent reports of the limited longterm durability of EVAR for treating AAA, we have found a survival advantage for those patients who undergo EVAR [4]. Our recent report has demonstrated that EVAR is an effective approach in which patients who undergo endovascular repair benefit from greater long-term survival, despite requiring more secondary procedures related to the aortic graft compared with their open counterparts. Additionally, those patients who undergo open AAA repair experience more nonvascular subsequent interventions, most commonly intra-abdominal or incision related complications [4]. These results contrast the conclusions from both the EVAR1 and DREAM trials in which the early survival advantage of EVAR diminished over time after development of complications requiring secondary intervention [7-9]. In the EVAR 1 Trial, there was no difference in aneurysm-related death after four years while there was no difference in mortality after one year in the DREAM Trial. This retrospective review examines mortality in patients who underwent secondary aortic procedures after initial EVAR or open infrarenal abdominal aortic aneurysm repair over a long-term surveillance.

Conrad et al. described their experience with secondary interventions after EVAR. Although their study included aortic as well as nonaortic secondary interventions, they described a secondary intervention rate of $11 \%$ in those patients who underwent EVAR. Their study encompassed 832 patients who underwent EVAR between 1999 and 2007. Thirty-day mortality for the patients requiring secondary intervention was $1.5 \%$ with an overall five-year survival of $70.3 \%$ at an average follow-up of 35 months and an average time to first reintervention of 17 months. An endovascular approach was performed in $71 \%$ of secondary interventions, including all procedures performed within the thirty-day postoperative period or for aneurysm-associated complications [8].

In contrast, our secondary aortic intervention rate was $6.6 \%(149 / 2256)$ for all patients who underwent infrarenal AAA repair, either EVAR or open. Common indications for secondary aortic procedure included reintervention for endoleak (specific for the endo first group), as well as elective repair of subsequent aortic aneurysmal degeneration, a common occurrence in the initial open repair groups. Sixtyfour percent of these aortic interventions (96/149) consisted of endovascular approaches, corresponding with the trend toward endovascular techniques for secondary interventions after EVAR or open AAA repair $[1,8,10]$. Supporting our data, Mehta et al. described outcomes of secondary procedures after EVAR in 1768 patients from 2002 to 2009. At a mean follow-up of thirty-four months, their overall mortality rate was $2.9 \%$ with $19.2 \%$ of patients requiring secondary aortic procedures, most of which were managed by endovascular approaches [10]. During our twenty-five-year study period, the time between aortic interventions averaged 6.2 years. Interestingly, further analysis revealed that the time between aortic interventions in Open- first patients ( 7 years in the Open-Open group, 13 years in the Open-Endo group) was more than double compared to that of Endo-first patients (3 years in the Endo-Endo group, 2 years in the EndoOpen group). This time interval difference may be related to the more frequent surveillance after EVAR and also for an increase in the vascular surgery presence in our region. Patients in the initial open repair group were younger than their initial EVAR counterparts; however, the age at secondary intervention was similar amongst all four groups. This finding may be due to the durability of open repair and the interval required for aortic degeneration and the development of secondary aortic aneurysms.

Mortality for each group after secondary intervention was also analyzed at varying time intervals. Thirty-day, threemonth, six-month, and one-year mortality for the EndoEndo group had been $3.3 \%, 8.3 \%, 10 \%$, and $15 \%$, respectively. For the Endo-Open group, mortality was $12 \%$ at thirty days, $18 \%$ at three and six months, and $27 \%$ at one year. Mortality for the Open-Endo patients at thirty days was $8.3 \%, 24 \%$ at three and six months, and $27 \%$ at one year. Lastly, for the Open-Open group, mortality at thirty days was $8.3 \%, 14 \%$ at three months, $19 \%$ at six months, and $22 \%$ at one year. These data likely reflect the invasiveness of the secondary aortic intervention as the highest morality was carried by the Endo-Open group which consisted of primarily patients who underwent explantation of a previously placed stent graft followed by aneurysm repair. In contrast, the patients in the Endo-Endo group carried the lowest short-term and longterm mortality, a reflection of the often less invasive catheterdirected strategies required during secondary intervention. While the Kaplan-Meier plot described in Figure 3 does suggest a separation of survival curves over the seventy-twomonth follow-up interval, the curves do appear to cross at twenty-four months, likely a reflection of Wilcoxon and log rank tests with $P$ values which are not statistically significant. However, as demonstrated in Table 2, the association of open secondary aortic interventions with long-term mortality revealed better survival amongst patients in the Endo-Endo and Endo-Open groups compared to those in the Open-Endo and Open-Open groups. Additionally, patients in the OpenEndo group had twofold greater likelihood of dying than their Endo-Endo counterparts. Therefore, the type of secondary intervention performed impacts short-term mortality while long-term mortality seems to be influenced by the initial aortic intervention.

Although we were able to follow patients for up to a total of eleven years, there were limitations to this retrospective study from a single academic institution database. Some patients may have been subsequently treated at other institutions and, hence, not captured by the registry. However, most patients who first have a procedure at our site return for any subsequent interventions due to regional practice patterns. We also included patients who had an initial aortic 
operation (endovascular or open) and were subsequently sent to our center for the secondary aortic procedure. This referral practice may create a lower than expected secondary intervention rate considering that we do not know the total number of patients treated at all the sites that may send patients to our center for their second operation. Furthermore, we did not include anatomic data (i.e., aneurysm sac size) which described the aortic conditions necessitating repair and which may have influenced the type of subsequent reintervention. Conrad et al. used multivariate analysis to define predictors of secondary intervention after EVAR which included aneurysm sac size greater than $5.5 \mathrm{~cm}$ and previous coil embolization [8]. As a result, further understanding of this data may promote tailoring an individualized approach to patients with aortic aneurysm disease based upon their anatomic information.

Over the past twenty-five years, the management of patients with infrarenal AAAs has evolved with advances in technology and clinical experience, benefiting overall patient outcomes. When EVAR first became commercially available, we more often selected patients who were medically at high risk and had appropriate anatomy for EVAR. As our experience grew and analysis of our early outcomes was performed [11], we became more liberal in its application to younger, healthier patients with appropriate anatomy or ill patients with marginal anatomic suitability. The data presented here depicts the natural history of patients who undergo AAA repair against the backdrop of an institution's paradigm shift toward selecting patients for endovascular repair based on anatomic variables rather than age or medical comorbidities. Over time, we have adopted a more aggressive EVAR-first approach to treating infrarenal AAAs.

\section{Conclusions}

Mortality in patients who undergo infrarenal AAA repair followed by secondary aortic intervention is associated with the initial operative approach, either endovascular or open. Long-term survival is greater amongst those patients who undergo initial EVAR, regardless of the type of secondary aortic intervention. As a result, survival amongst patients with infrarenal AAAs may be improved by an EVAR-first algorithm.

\section{Disclosure}

This paper was presented during Scientific Session IV, Southern Association for Vascular Surgery 36th Annual Meeting, January 18-21, 2012, Scottsdale, Arizona.

\section{Conflict of Interests}

The authors declare that there is no conflict of interests regarding the publication of this paper.

\section{References}

[1] J.-P. Becquemin, L. Kelley, T. Zubilewicz et al., "Outcomes of secondary interventions after abdominal aortic aneurysm endovascular repair," Journal of Vascular Surgery, vol. 39, no. 2, pp. 298-305, 2004.

[2] N. V. Dias, L. Riva, K. Ivancev, T. Resch, B. Sonesson, and M. Malina, "Is there a benefit of frequent CT follow-up after EVAR?" European Journal of Vascular and Endovascular Surgery, vol. 37, no. 4, pp. 425-430, 2009.

[3] R. J. F. Laheij, J. Buth, P. L. Harris, F. L. Moll, W. J. Stelter, and E. L. G. Verhoeven, "Need for secondary interventions after endovascular repair of abdominal aortic aneurysms. Intermediate-term follow-up results of a European collaborative registry (EUROSTAR)," British Journal of Surgery, vol. 87, no. 12, pp. 1666-1673, 2000.

[4] B. E. Quinney, G. M. Parmar, S. B. Nagre et al., "Long-term single institution comparison of endovascular aneurysm repair and open aortic aneurysm repair," Journal of Vascular Surgery, vol. 54, no. 6, pp. 1592-1598, 2011.

[5] M. F. Conrad and R. P. Cambria, "Contemporary management of descending thoracic and thoracoabdominal aortic aneurysms: endovascular versus open," Circulation, vol. 117, no. 6, pp. 841-852, 2008.

[6] T. A. Abbruzzese, C. J. Kwolek, D. C. Brewster et al., "Outcomes following endovascular abdominal aortic aneurysm repair (EVAR): an anatomic and device-specific analysis," Journal of Vascular Surgery, vol. 48, no. 1, pp. 19-28, 2008.

[7] J. D. Blankensteijn, S. E. de Jong, M. Prinssen et al., "Twoyear outcomes after conventional or endovascular repair of abdominal aortic aneurysms," The New England Journal of Medicine, vol. 352, no. 23, pp. 2398-2405, 2005.

[8] M. F. Conrad, A. B. Adams, J. M. Guest et al., "Secondary intervention after endovascular abdominal aortic aneurysm repair," Annals of Surgery, vol. 250, no. 3, pp. 383-389, 2009.

[9] "Endovascular aneurysm repair versus open repair in patients with abdominal aortic aneurym (EVAR trial 1): randomised controlled trial," The Lancet, vol. 365, no. 9478, pp. 2179-2186, 2005.

[10] M. Mehta, Y. Sternbach, J. B. Taggert et al., "Long-term outcomes of secondary procedures after endovascular aneurysm repair," Journal of Vascular Surgery, vol. 52, no. 6, pp. 1442-1448, 2010.

[11] W. D. Jordan, F. Alcocer, D. J. Wirthlin et al., "Abdominal aortic aneurysms in 'high-risk' surgical patients: comparison of open and endovascular repair," Annals of Surgery, vol. 237, no. 5, pp. 623-630, 2003. 


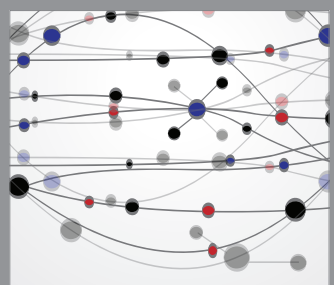

The Scientific World Journal
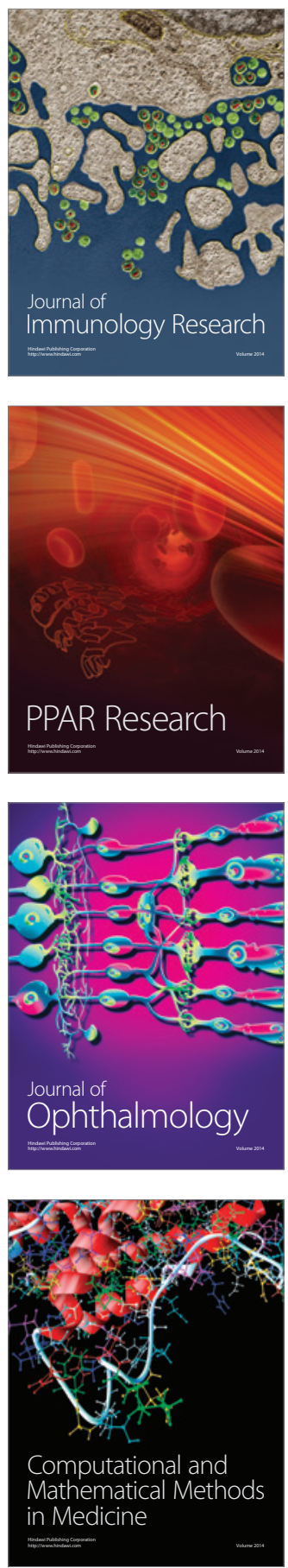

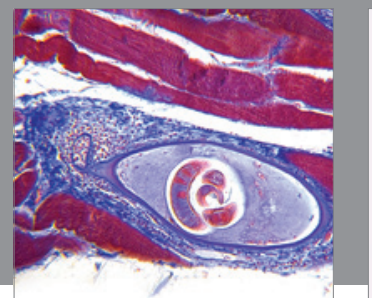

Gastroenterology

Research and Practice
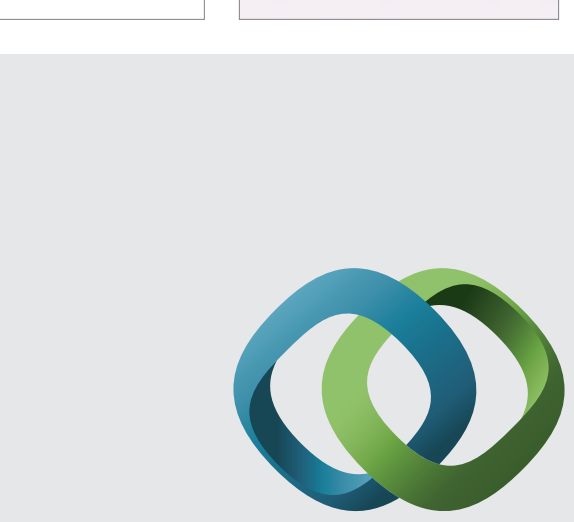

\section{Hindawi}

Submit your manuscripts at

http://www.hindawi.com
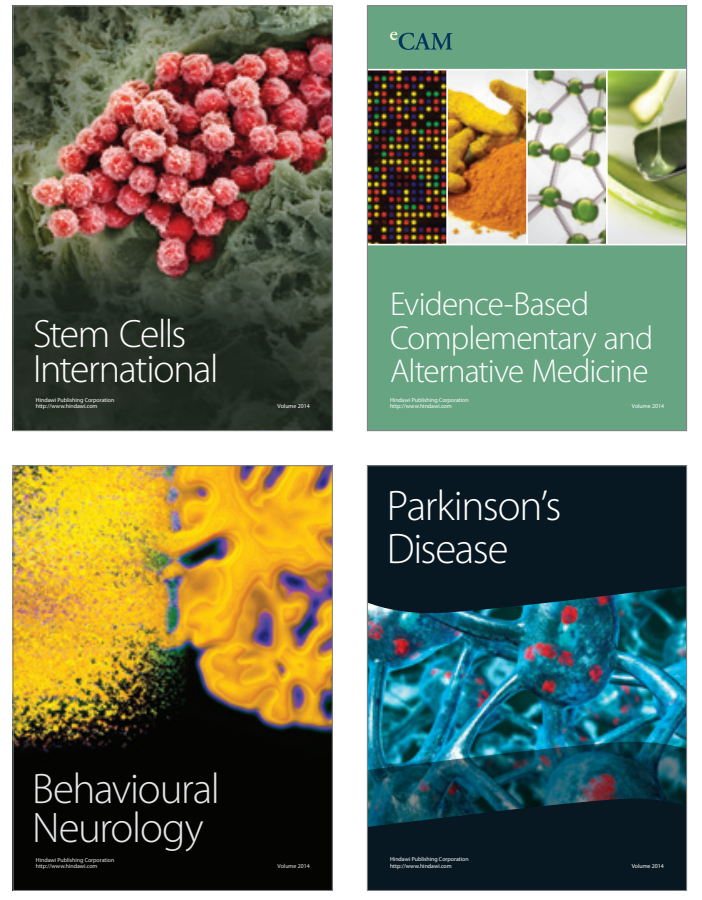
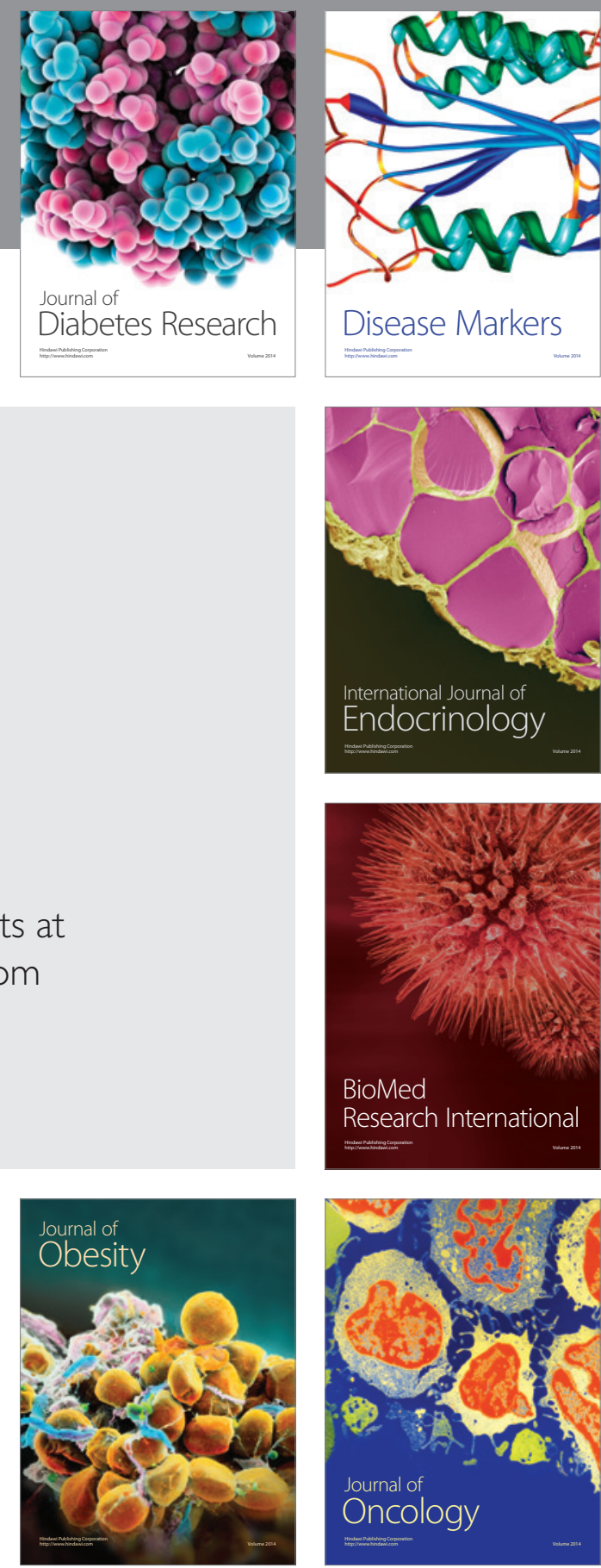

Disease Markers
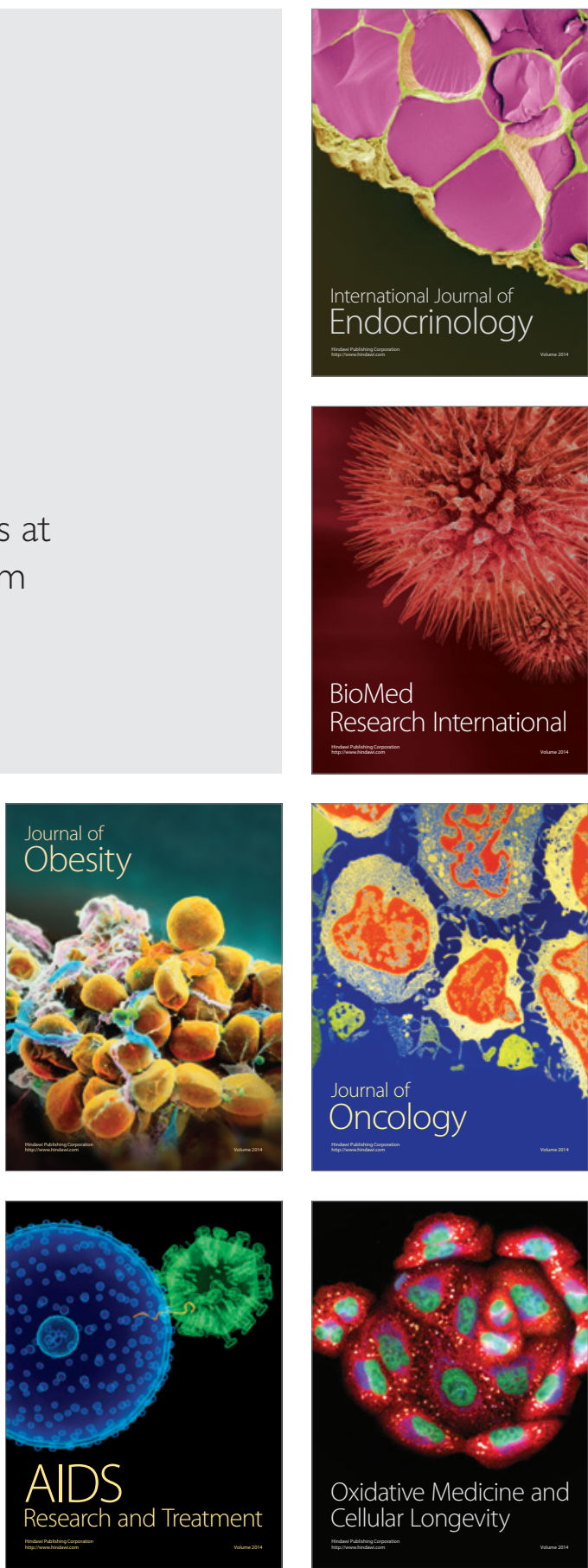